\title{
Bimetallic $\mathrm{Cu} / \mathrm{Rh}$ Catalyst for Preferential Oxidation of CO in $\mathrm{H}_{2}$ : a DFT Study
}

Chuwei Zhu, ${ }^{\dagger}$ Xiang-Kui Gu* ${ }^{*}+$ and Wei-Xue $\mathrm{Li}^{*}{ }^{\dagger}, \S$

†Department of Chemical Physics, School of Chemistry and Materials Science, University of Science and Technology of China, Hefei, Anhui 230026, China

${ }^{\ddagger}$ School of Power and Mechanical Engineering, Wuhan University, Wuhan 430072,

China

${ }^{\S}$ Hefei National Laboratory for Physical Science at the Microscale, University of

Science and Technology of China, Hefei, Anhui 230026, China 


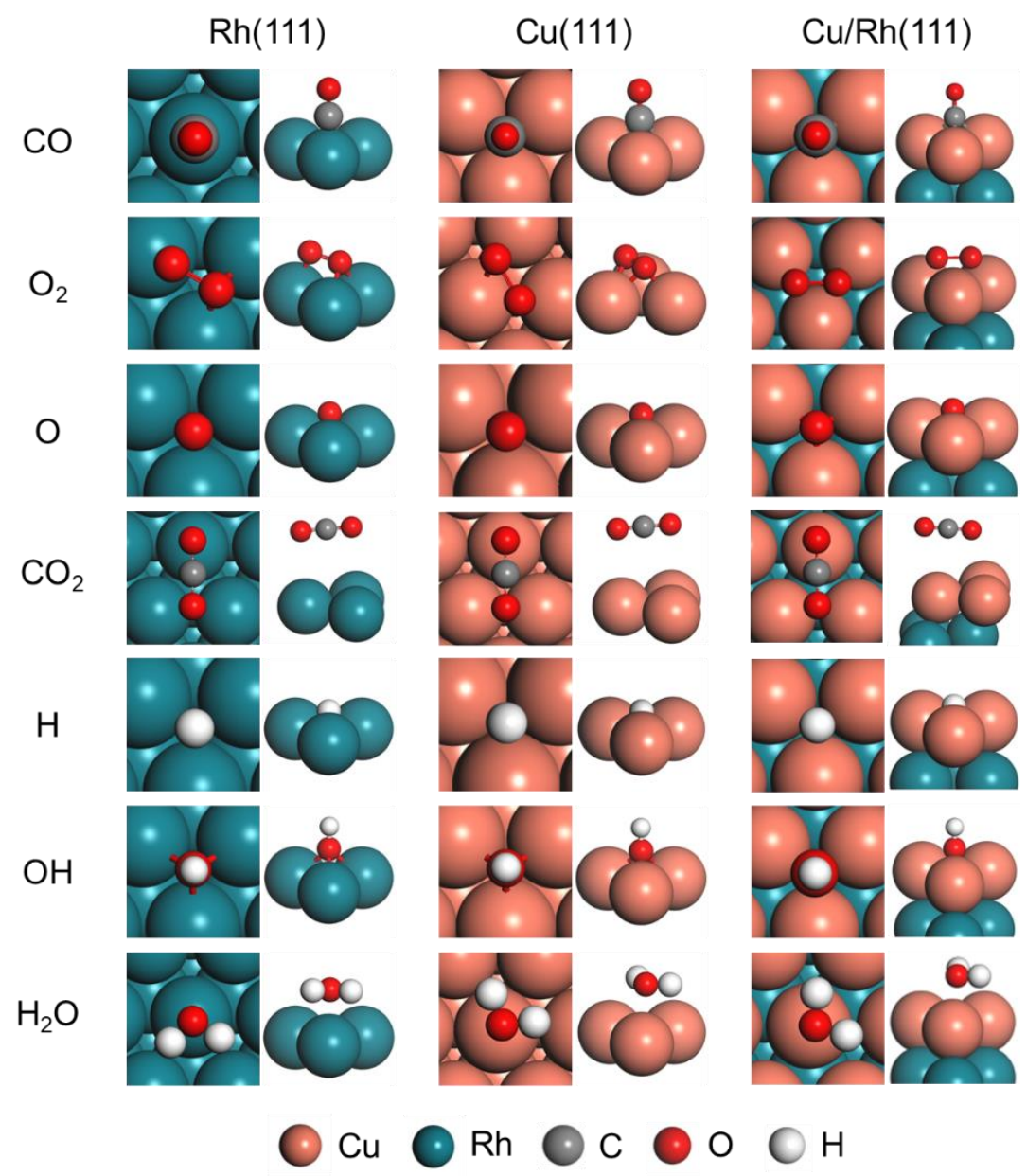

Figure S1. Optimized geometries of the most stable adsorption states of reactants and intermediates involved in PROX reaction on $\mathrm{Rh}(111), \mathrm{Cu}(111)$ and $\mathrm{Cu} / \mathrm{Rh}(111)$ surfaces. The cyan, orange, gray, red, and white spheres represent $\mathrm{Rh}, \mathrm{Cu}, \mathrm{C}, \mathrm{O}$, and $\mathrm{H}$ atoms, respectively. 

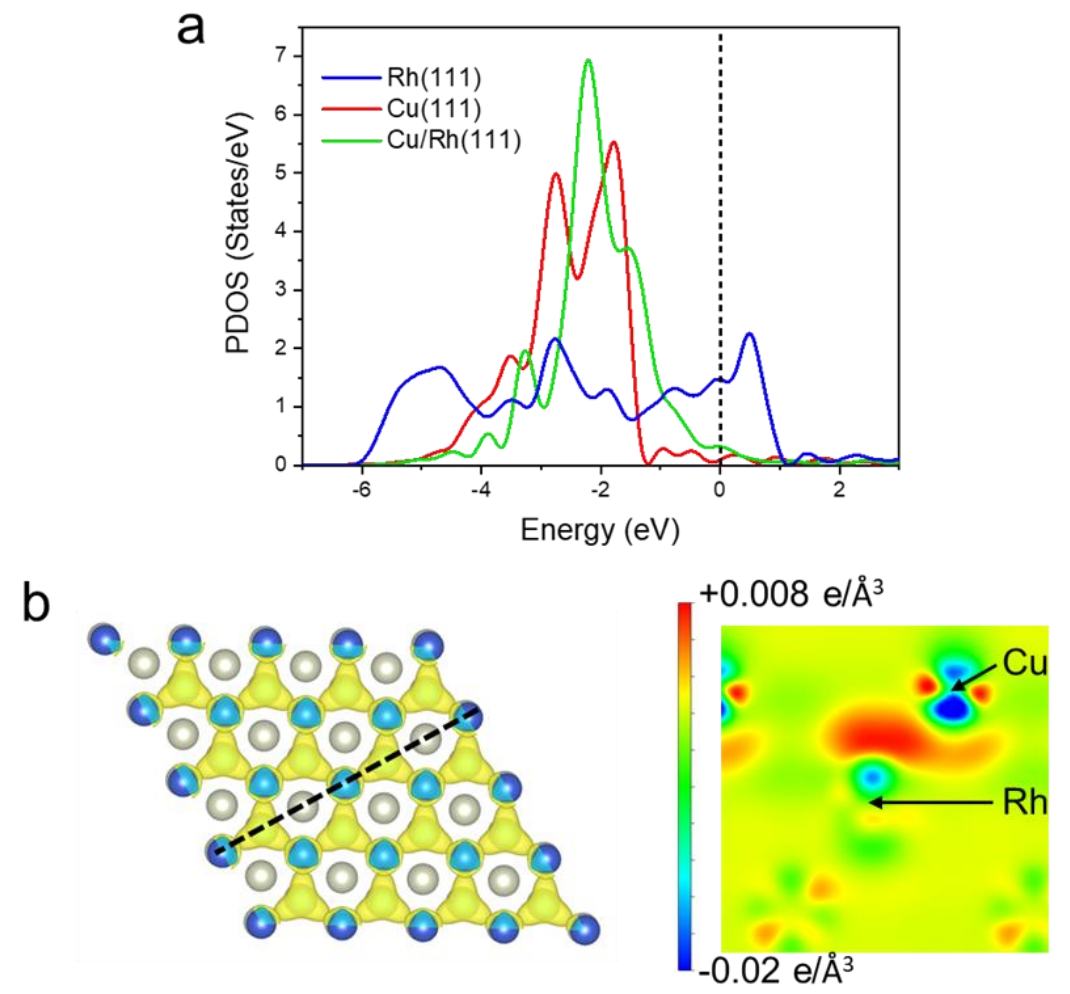

Figure S2. a) Projected density of states for the $d$-orbital of a surface atom on $\mathrm{Rh}(111)$, $\mathrm{Cu}(111)$ and $\mathrm{Cu} / \mathrm{Rh}(111)$ surfaces. b) Contour plot and its $2 \mathrm{D}$ section of charge density difference between $\mathrm{Cu}$ overlayer and $\mathrm{Rh}$ substrate in bimetallic $\mathrm{Cu} / \mathrm{Rh}(111)$. The blue and grey spheres represent $\mathrm{Cu}$ and $\mathrm{Rh}$ atoms, respectively. The areas of cyan and yellow contours represent the depletion and accumulation of electrons in a unit of $0.005 \mathrm{e} / \AA^{3}$, respectively. 


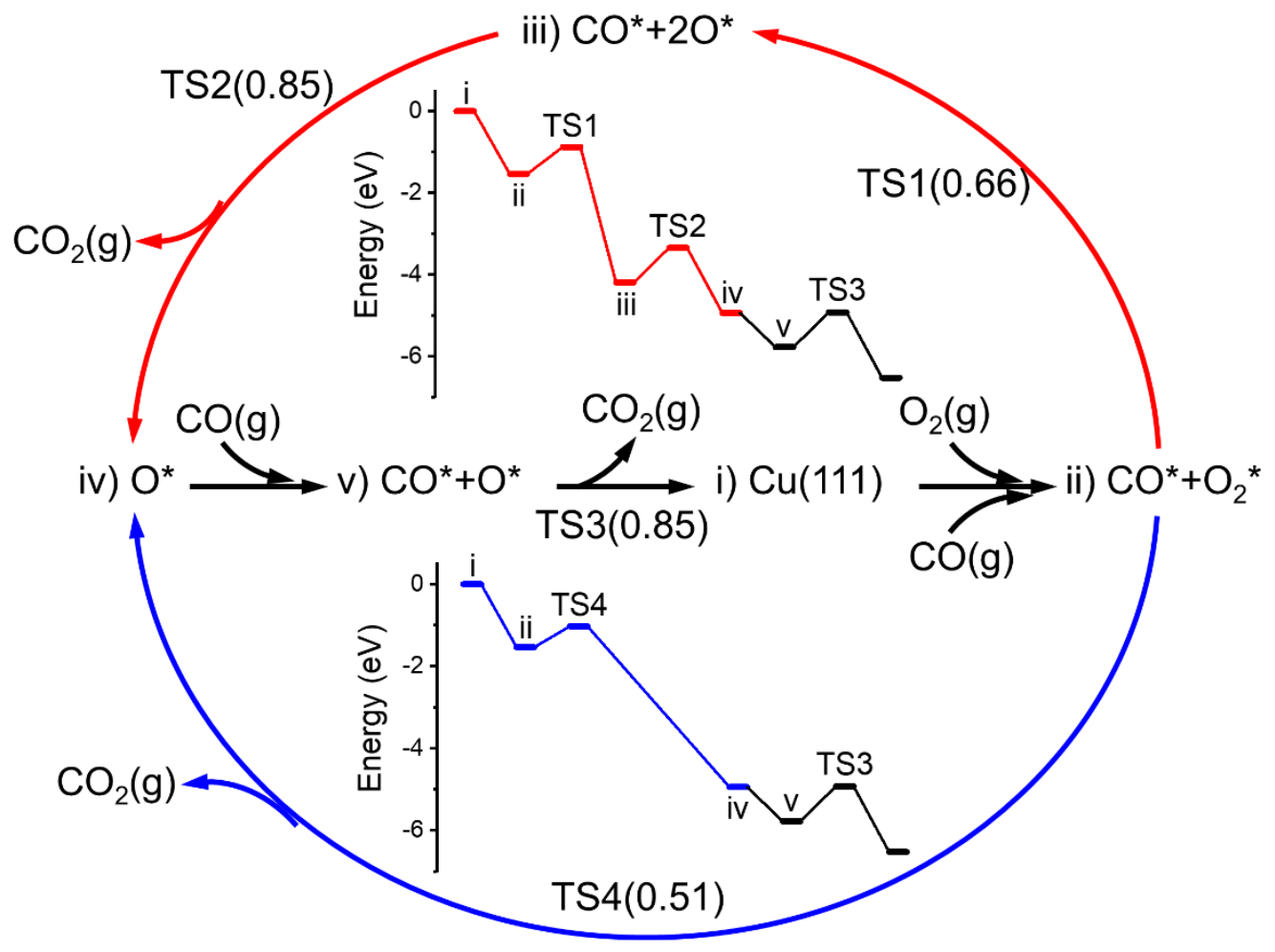

Figure S3. Potential energy profiles and catalytic cycles for $\mathrm{CO}$ oxidation via dissociative (red lines) and associative mechanisms (blue lines) on $\mathrm{Cu}(111)$. 


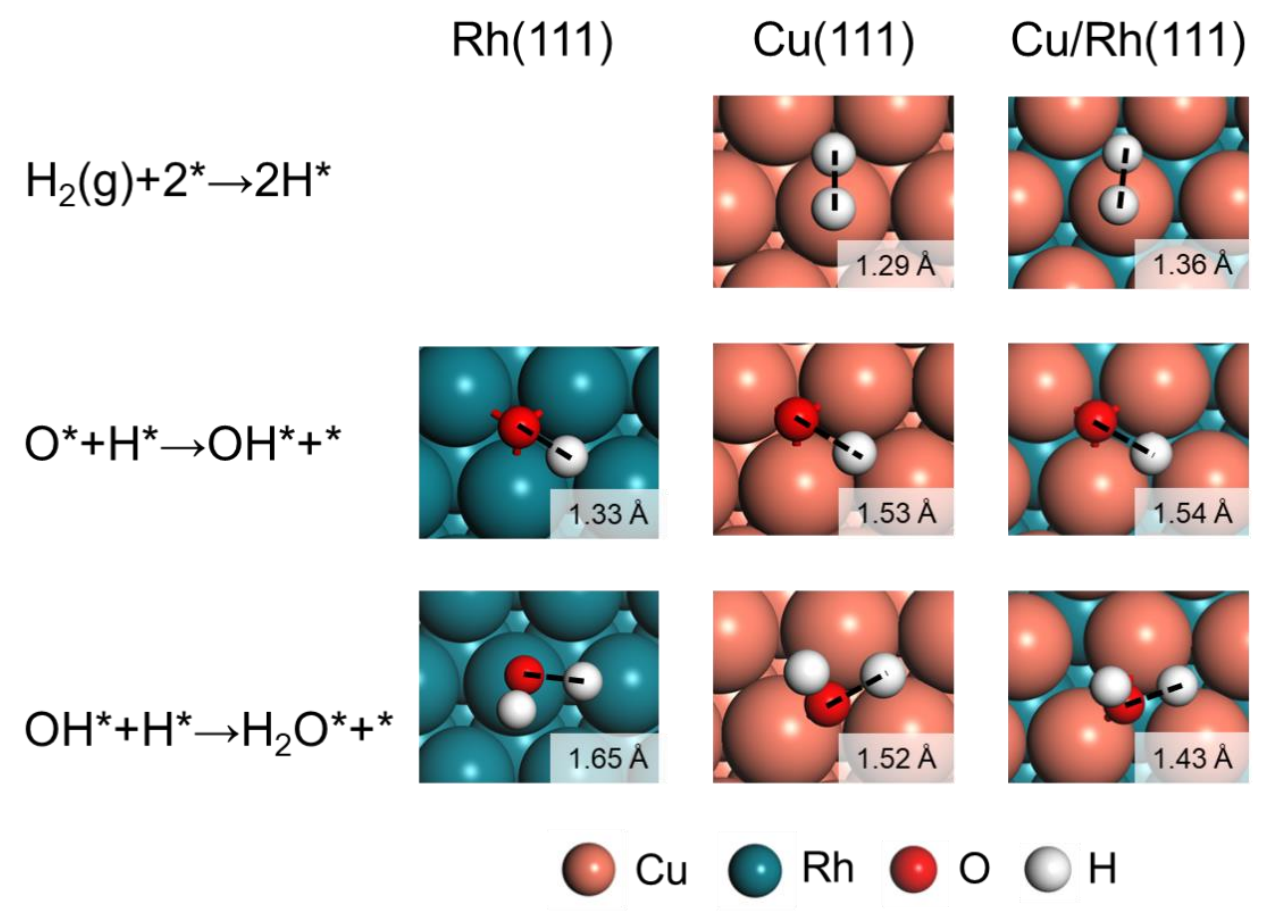

Figure S4. Optimized structures of transition states in $\mathrm{H}_{2}$ oxidation on $\mathrm{Rh}(111), \mathrm{Cu}(111)$ and $\mathrm{Cu} / \mathrm{Rh}(111)$ surfaces. 

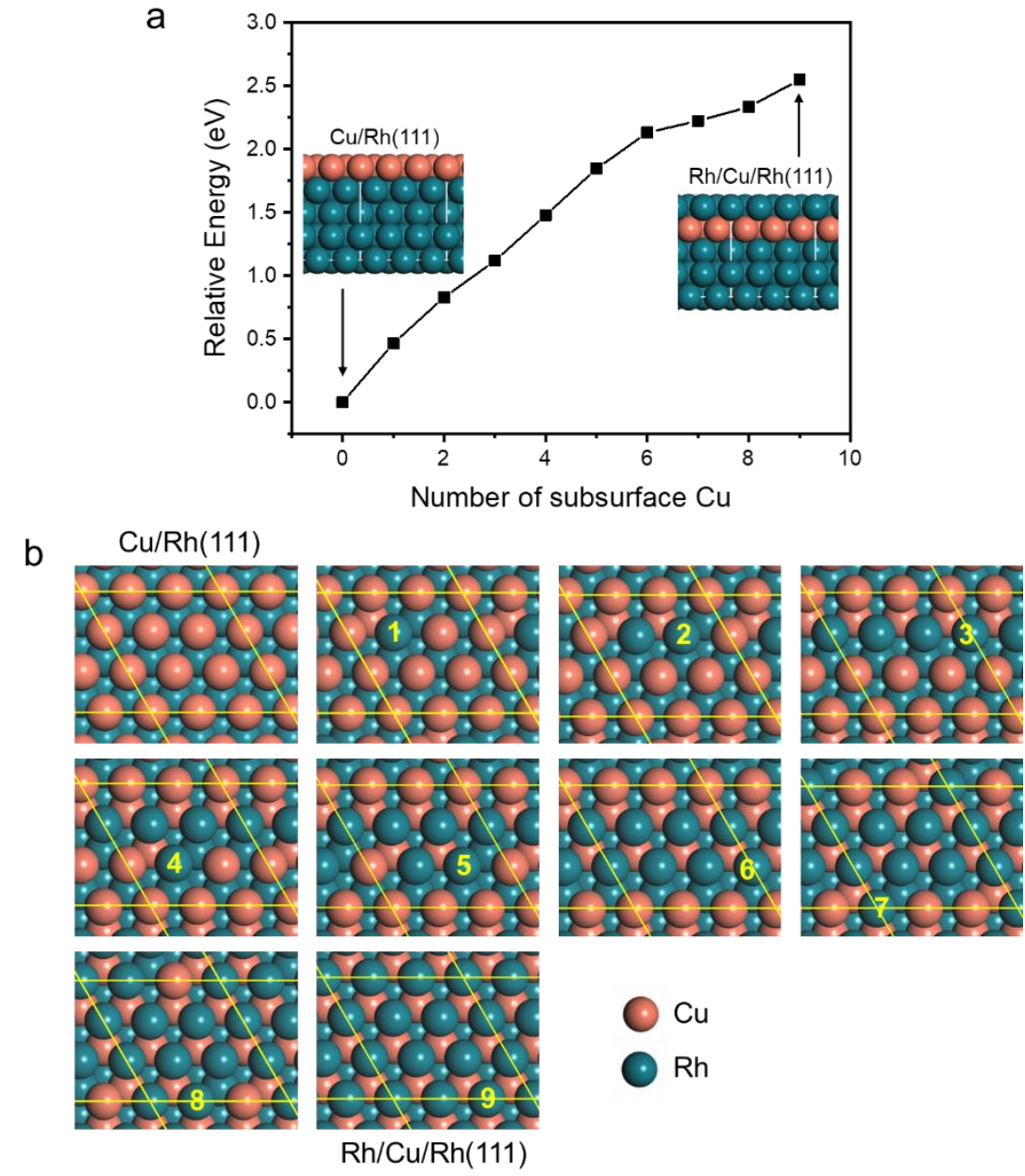

Figure S5. a) Energy variation of a $(3 \times 3)-\mathrm{Cu} / \mathrm{Rh}(111)$ slab when exchanging surface $\mathrm{Cu}$ and subsurface $\mathrm{Rh}$ one after another and $\mathrm{b}$ ) top views of the corresponding structures. 
a

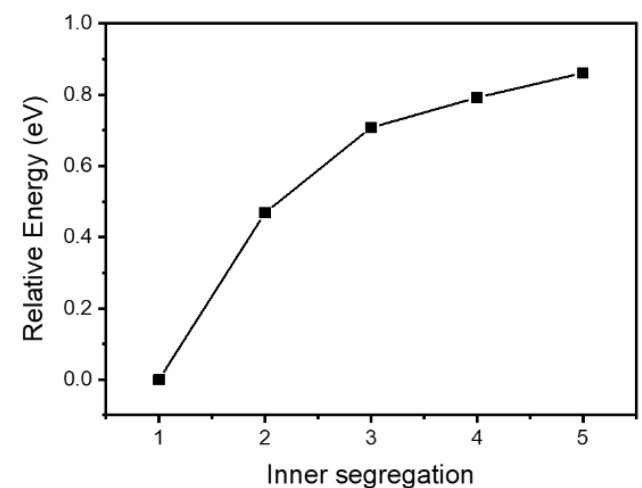

b

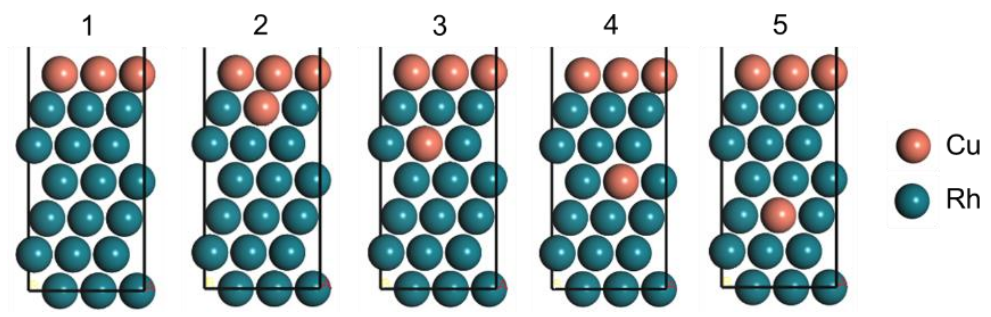

Figure S6. a) Energy variation of a $\mathrm{Cu} / \mathrm{Rh}(111)$ slab with diffusion of one surface $\mathrm{Cu}$ atom into inner region and b) side views of the corresponding structures. This calculation is based on a thicker slab with 6-layer $\mathrm{Rh}$ substrate and a $\mathrm{Cu}$ monolayer. 


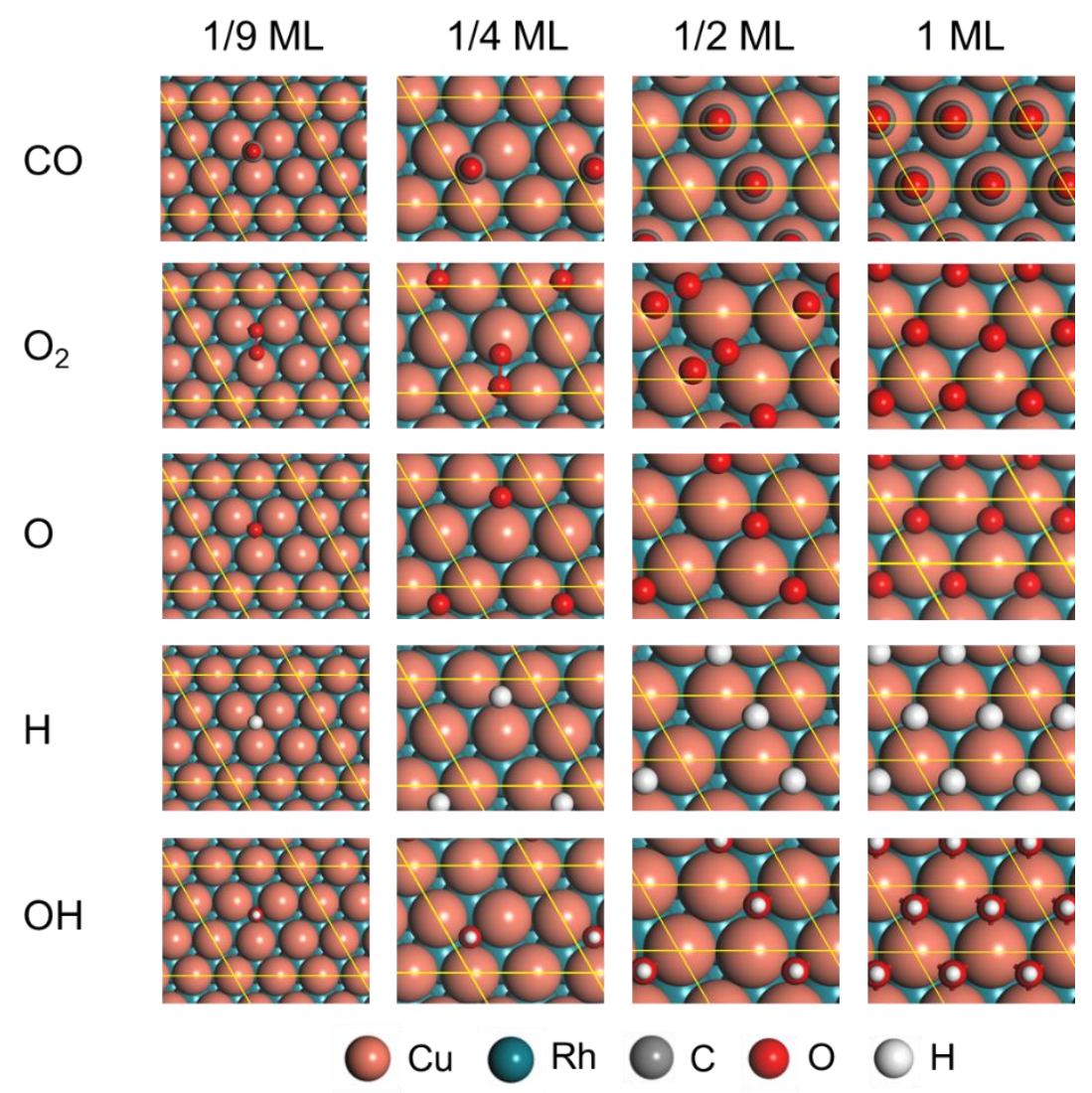

Figure S7. Optimized geometries of adsorbed $\mathrm{CO}, \mathrm{O}_{2}, \mathrm{O}, \mathrm{H}$ and $\mathrm{OH}$ on $\mathrm{Cu} / \mathrm{Rh}(111)$ surface at different coverages. 
a
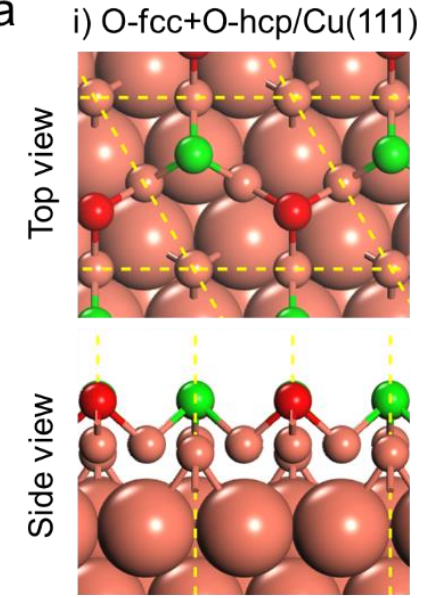

b i) O-fcc+O-hcp/CuRh(111)
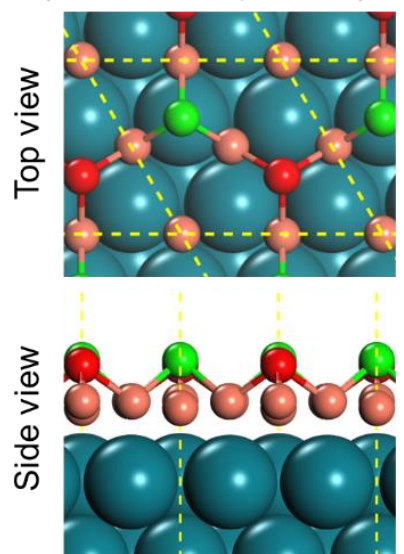

ii) $\mathrm{Cu}_{2} \mathrm{O} / \mathrm{Cu}(111)$
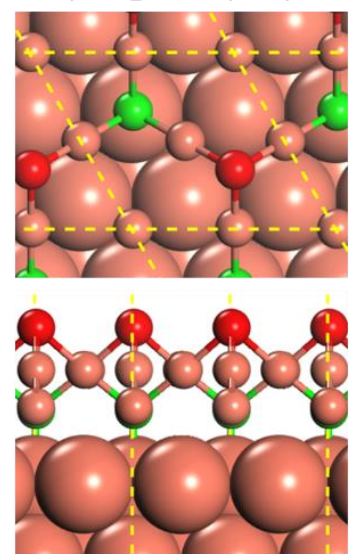

ii) $\mathrm{Cu}_{2} \mathrm{O} / \mathrm{Rh}(111)$
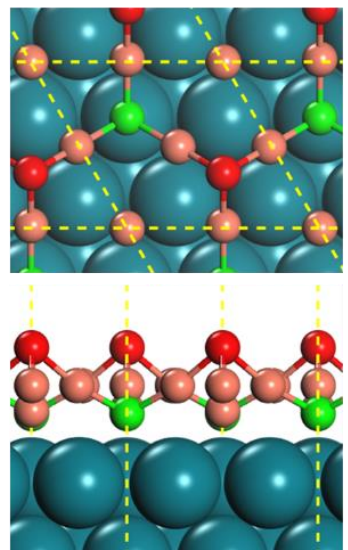

iii) $\mathrm{Cu}_{4} \mathrm{O}_{1} / \mathrm{Cu}(111)$
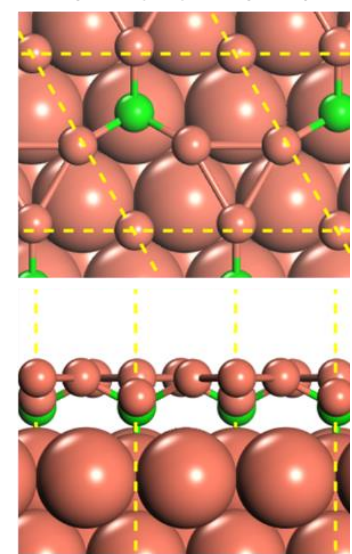

iii) $\mathrm{Cu}_{4} \mathrm{O}_{1} / \mathrm{Rh}(111)$
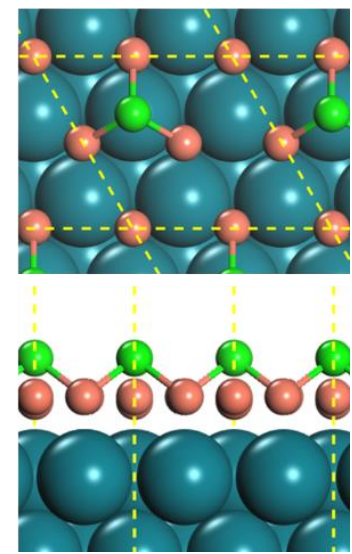

Figure S8. Optimized structures of coadsorbed $\mathrm{O}$ atoms, $\mathrm{Cu}_{2} \mathrm{O}$ overlayer, and partially reduced $\mathrm{Cu}_{4} \mathrm{O}_{1}$ overlayer on a) $\mathrm{Cu}(111)$ and b) $\mathrm{Cu} / \mathrm{Rh}(111)$. The orange, cyan, green and red spheres represent $\mathrm{Cu}$ atom, $\mathrm{Rh}$ atom, oxygen atom at hep site (or in the lower planes of $\mathrm{Cu}_{2} \mathrm{O}$ ) and fcc site (or in the upper plane of $\mathrm{Cu}_{2} \mathrm{O}$ ), respectively. 
Table S1. Activation barriers $\left(E_{\mathrm{a}}\right)$ and reaction energies $\left(E_{\mathrm{r}}\right)$ of elementary reactions involved in $\mathrm{PROX}$ on $\mathrm{Rh}(111), \mathrm{Cu}(111)$ and $\mathrm{Cu} / \mathrm{Rh}(111)$ surfaces.

\begin{tabular}{ccccccc}
\hline \multirow{2}{*}{$\begin{array}{c}\text { Elementary } \\
\text { reaction }\end{array}$} & \multicolumn{2}{c}{$\mathrm{Rh}(111)$} & \multicolumn{2}{c}{$\mathrm{Cu}(111)$} & \multicolumn{2}{c}{$\mathrm{Cu} / \mathrm{Rh}(111)$} \\
\cline { 2 - 7 } & $E_{\mathrm{a}}(\mathrm{eV})$ & $E_{\mathrm{r}}(\mathrm{eV})$ & $E_{\mathrm{a}}(\mathrm{eV})$ & $E_{\mathrm{r}}(\mathrm{eV})$ & $E_{\mathrm{a}}(\mathrm{eV})$ & $E_{\mathrm{r}}(\mathrm{eV})$ \\
\hline 1 & 0.15 & -2.74 & 0.12 & -2.66 & 0.14 & -2.79 \\
2 & 0.26 & -2.74 & 0.66 & -2.66 & 0.77 & -2.79 \\
3 & 1.44 & 0.82 & 0.85 & -0.75 & 0.97 & -0.26 \\
5 & - & - & 0.51 & -3.41 & 0.58 & -3.04 \\
6 & 0.00 & -1.11 & 0.61 & -0.42 & 0.39 & -0.69 \\
7 & 1.29 & 0.36 & 1.14 & -0.41 & 1.17 & -0.44 \\
\hline
\end{tabular}

These elementary reactions are:

1. $\mathrm{O}_{2} *+* \rightarrow 2 \mathrm{O} *(\mathrm{w} / \mathrm{o} \mathrm{CO})$

2. $\mathrm{O}_{2}{ }^{*} * \rightarrow 2 \mathrm{O} *$ (with $\mathrm{CO}$ coadsorption)

3. $\mathrm{CO} *+\mathrm{O} * \rightarrow \mathrm{CO}_{2}(\mathrm{~g})+*$

4. $\mathrm{CO}^{*}+\mathrm{O}_{2} * \rightarrow \mathrm{CO}_{2}(\mathrm{~g})+\mathrm{O} *+*$

5. $\mathrm{H}_{2}(\mathrm{~g})+2 * \rightarrow 2 \mathrm{H}^{*}$

6. $\mathrm{O}^{*}+\mathrm{H}^{*} \rightarrow \mathrm{OH}^{*+*}$

7. $\mathrm{OH}^{*+}+\mathrm{H}^{*} \rightarrow \mathrm{H}_{2} \mathrm{O}^{*+*}$ 